\title{
Comportements sexuels et SIDA
}

L'Organisation Mondiale de la Santé (OMS) estime que depuis le début de l'épidémie de Sida, environ 13 millions de personnes ont été infectées par le virus de l'immunodéficience humaine (VIH) dans le monde. La mise au point d'un vaccin efficace nécessitera encore de nombreuses années, si elle peut être menée à bien. La prévention est dans ces conditions un élément fondamental pour lutter contre le développement de l'épidémie. On estime qu'environ $70 \%$ des personnes infectées dans le monde l'ont été par des contacts hétérosexuels, $15 \%$ par des contacts homosexuels, la transmission par voie veineuse étant minoritaire.

Dans les pays développés, bien que la transmission parmi les homosexuels soit largement majoritaire (un peu moins de $50 \%$ des cas de SIDA en France), les contacts hétérosexuels sont à l'origine d'une proportion croissante de cas. Ce sont en particulier, les femmes qui constituent le groupe de la population dans lequel l'épidémie croît le plus rapidement, la transmission par voie sanguine étant également importante parmi elles. Devant cette situation épidémiologique, l'étude des comportements sexuels et la prévention des risques liés aux comportements sexuels est une tâche prioritaire. Les efforts déjà réalisés dans ce sens ont été d'importance limitée en dehors de la communauté homosexuelle (la promotion du safer sex) et leur orientation a souvent été empirique. Par exemple, la plupart des messages utilisés ont été élaborés sans disposer d'informations précises sur les comportements sexuels effectifs de la population. A de très rares exceptions près, ces messages n'ont jamais pris en compte des différences entre hommes et femmes, ou n'ont pas suffisamment été élaborés en considérant les conditions psycho-sociales, culturel- les et économiques des personnes auxquelles ils étaient adressés.

Deux importantes études portant sur la connaissance des comportements sexuels de la population générale viennent d'être réalisées, l'une en France [1], la seconde en GrandeBretagne [2]. Ces études ont pour but d'apporter des informations utiles à l'élaboration et à l'évaluation des stratégies de prévention des maladies sexuellement transmissibles (MST et SIDA), et de permettre une meilleure prévision du développement de l'épidémie.

L'étude française a débuté en juillet 1989 à l'initiative de l'Agence Nationale de Recherche sur le SIDA (ANRS), elle comporte trois volets : 1. une enquête sur les personnes adultes âgées de 18 à 69 ans résidant en France métropolitaine;

2. une enquête sur les personnes adultes âgées de 18 à 69 ans résidant dans les départements français d'Amérique (Guyane, Martinique, Guadeloupe) ; 3. une enquête sur les adolescents âgés de 15 à 18 ans résidant en France métropolitaine.

Ces recherches sont menées par un groupe de 25 chercheurs de l'INSERM, de l'INED, du CNRS et d'universités parisiennes (Paris VI, VII, XI et XIII). L'enquête adulte a porté sur un échantillon de 20055 personnes, interrogées par téléphone par 110 enquêteurs des instituts de sondage BVA et MV2. Des précautions méthodologiques très importantes ont été prises pour garantir la plus grande fiabilité des réponses. Le taux d'acceptation de l'enquête a été de $77 \%$ parmi les personnes contactées, seulement $3 \%$ des personnes qui ont débuté le questionnaire ont interrompu l'enquête avant la fin.

Les premiers résultats concernent la description des comportements sexuels et le recours aux méthodes de protection contre le risque de transmission de MST et de SIDA.

L'âge au premier rapport sexuel (17 ans pour les hommes et 18 ans pour les femmes) ne s'est pas modifié depuis 20 ans et ne semble donc pas avoir été affecté par la survenue de l'épidémie de SIDA.

Le nombre de partenaires sexuels au cours des 12 derniers mois s'établit en moyenne à 1,32 pour les hommes et 1,06 pour les femmes, la proportion de sujets multipartenaires - c'est-à-dire ayant eu au moins deux partenaires sexuels durant l'année écoulée - étant de $13,8 \%$ parmi les hommes et 6,3 \% parmi les femmes. Le multipartenariat varie fortement avec l'âge : à 18-19 ans il est de 38,4\% pour les hommes et $18,5 \%$ pour les femmes, il diminue progressivement à partir de 25 ans. L'analyse du nombre de partenaires au moment de l'enquête montre que le multipartenariat des jeunes est davantage un multipartenariat sériel (un partenaire puis un autre) alors que le multipartenariat des groupes plus âgés est un multipartenariat simultané (plusieurs partenaires en même temps). La fréquence moyenne des rapports sexuels (2 par semaine pour les hommes et 1,75 pour les femmes) est très voisine de celle enregistrée il y a 20 ans [3].

Une évolution importante concerne les pratiques sexuelles déclarées. La masturbation est rapportée par $42 \%$ des femmes et $84 \%$ des hommes, les pratiques bucco-génitales (fellation et cunilinctus) par $70 \%$ à $80 \%$ des personnes interrogées environ, enfin la pénétration anale par $24 \%$ des femmes et $30 \%$ des hommes. L'augmentation de déclaration de ces pratiques depuis 20 ans peut traduire à la fois une réelle 
augmentation de leur réalisation et une plus grande facilité à les déclarer aujourd'hui, c'est-à-dire une meilleure acceptation sociale.

L'homosexualité, définie comme étant la pratique au moins une fois de rapports sexuels avec une personne du même sexe, a concerné au cours de la vie $4,1 \%$ des hommes et $2,6 \%$, des femmes, ces chiffres étant respectivement de $1,4 \%$ et $0,4 \%$ pour les 5 dernières années et $1,1 \%$ et $0,3 \%$ pour les 12 derniers mois. La grande majorité de ces personnes sont bisexuelles (82\% des hommes et $78 \%$ des femmes). Les variations sont importantes selon l'âge et le type d'agglomération, 4,6\% des hommes de 45-49 ans habitant l'agglomération parisienne ayant par exemple eu des rapports homosexuels dans l'année écoulée.

Le recours à la prostitution ne concerne que $3,3 \%$ des hommes pour les cinq dernières années, l'évolution la plus importante concernant la très importante diminution du recours à la prostitution lors du premier rapport sexuel $(10 \%$ il y a 20 ans, $2 \%$ aujourd'hui).

Entre 20 et 34 ans, près de $7 \%$ des femmes disent avoir subi des rapports sexuels imposés sous la contrainte. La période la plus dangereuse en ce qui concerne ce type de sévices est celle des débuts de l'adolescence. Ces rapports sexuels imposés se sont produits dans un cas sur quatre avec un inconnu. Mais dans plus d'un cas sur deux, il s'agissait d'une personne connue mais étrangère à la famille et dans un cas sur cinq d'un membre de la famille.

L'un des objectifs de cette enquête est de pouvoir analyser, de façon fine, le recours aux précautions contre le risque de transmission de MST, et en particulier l'utilisation de préservatifs. Au total $31 \%$ des hommes et $22 \%$ des femmes (parmi les sexuellement actifs) ont utilisé un préservatif au moins une fois au cours des 12 derniers mois; c'est le cas d'environ $80 \%$ des hommes de 18-19 ans et $50 \%$ des femmes du même âge, le recours diminuant ensuite nettement avec l'âge.

Le niveau de protection dans la population potentiellement exposée au risque de transmission est élevé, puisque parmi les sujets âgés de 18 à 44 ans, trois-quarts des hommes homobisexuels, $65 \%$ des hommes et la moitié des femmes multipartenaires hétérosexuels ont utilisé le préservatif au moins 1 fois aux cours de l'année écoulée. Cependant, la proportion de ces personnes qui ne se sont à aucun moment protégées par ce moyen reste encore trop importante pour enrayer le développement de l'épidémie. Les facteurs les plus importants liés à l'utilisation du préservatif sont l'âge, le type de partenaire, la durée de la relation et la perception qu'un sujet peut avoir de son propre risque de contracter le Sida. Il apparaît cependant que, parmi les multipartenaires, une proportion importante de ceux qui utilisent le préservatif se sentent protégés et donc moins à risque d'être contaminés. Cela met en évidence l'existence possible, dans ce groupe, d'une diminution de la vigilance face au développement toujours présent de l'épidémie, et la nécessité de maintenir des politiques de prévention dynamiques.

L'analyse entreprise actuellement tente de décrire quelles sont les caractéristiques démographiques, sociologiques et psychologiques des personnes qui ont adopté des méthodes de protection contre les MST et le SIDA, et d'analyser les logiques de protection (diminution du nombre de partenaires sexuels, utilisation de méthodes barrières, en particulier les préservatifs, ou enfin l'abandon de pratiques à risque) de façon à aider à une meilleure définition des stratégies de prévention.

Les résultats bruts observés dans cette étude sont très voisins des premiers résultats disponibles de l'enquête britannique. Par exemple, les taux de multipartenariat et d'homosexualité masculine sont pratiquement superposables dans les deux études. En ce qui concerne l'utilisation du préservatif masculin ou la pratique du test de dépistage pour le VIH, des différences semblent exister. Une analyse détaillée comparative des deux études va maintenant être entreprise, son but étant de tenter de comprendre quels sont les facteurs qui pourraient expliquer que, bien que la taille, la structure démographique et le type de comportements de ces deux pays soient si proches, le nombre cumulé de cas de Sida depuis le début de l'épidémie est environ 4 fois plus important en France qu'en Grande-Bretagne.

L'étude française a pû être réalisée grâce à un financement important (7 millions de francs environ) des pouvoirs publics. L'enquête britannique, après le veto de Mme. Thatcher, a été financée par la fondation Wellcome (1 million de livres). Quant au projet américain équivalent, les refus réitérés de l'administration Bush en ont rendu, pour le moment, la réalisation impossible.

Il est notable que, pour une fois, les européens ont pû faire preuve d'initiatives leur permettant de se doter rapidement d'outils d'action dans la prévention du SIDA et plus généralement de connaissances dans le domaine complexe de la sexualité humaine.

\section{Alfred Spira Nathalie Bajos ACSF* $^{*}$}

1. ACSF Investigators AIDS and sexual behaviour. Nature 1992; 360 : 407-9.

2. Johnson A, Wadsworth J, Wellings K, Bradshaw S, Field J. Sexual lifestyles and HIV risk. Nature, 1992 ; 360 : 410-2.

3. Simon P, Gondonneau J, Mironer I, DourlenRollier AM. Rapport sur le comportement sexuel des français. Paris : R. Julliard et P. Chanon, 1972

- ACSF : Analyse des componkements sexuels en France, groupe de chercheurs composé de: A. Spira, Directeur, N. Bajos, coordinatrice, $A$. Béin, N. Beltzer, $M$. Bozon, B. Ducot, A. Durandeau, A. Fernand, A Giami, M. Giraud, A. Gilloire, H. Leridon, D. Lud wig, A. Messiah, JP. Moatti, L. Mounnier, H. Olomucki, J. de Poplavsky, B. Riandey, R. Spencer, J.M. Sztalryd, H. Touzard. 